\title{
ANÁLISIS COMPARATIVO DE LOS CENTROS PÚBLICOS Y PRIVADOS MEDIDO A TRAVÉS DEL PREMIO EXTRAORDINARIO DE LA ESO ${ }^{1}$ EN GALICIA
}

\author{
Francisco Jesús Ferreiro Seoane \\ Universidad de Santiago de Compostela
}

\begin{abstract}
RESUMEN: El objetivo del presente artículo es analizar la influencia de la titularidad del centro y del entorno familiar en el rendimiento académico medido a través de las pruebas del premio extraordinario de la ESO. En primer lugar, se hace un repaso de la literatura que permita conocer la aportación a otros autores respecto a la influencia de los centros u otras variables en el rendimiento del alumnado. Posteriormente a partir de las calificaciones en todas las asignaturas (8) de todos los discentes (1.522) presentados al premio extraordinario de la ESO para el período 2008-2013, se han analizado dichos resultados clasificándolos según la titularidad del centro aplicando el Análisis Unifactorial. Además, se analizó en qué medida existe una influencia del entorno familiar en dichos resultados, medidos a través de la formación de los padres y de su poder adquisitivo. Los principales resultados son que no existen diferencias significativas en relación a la titularidad de los centros excepto en "CSGH"2 y "Lengua Extranjera". Además, se constata la influencia del entorno familiar en el rendimiento académico, si bien en "Inglés" se mantiene la a favor de titularidad privada cuando se hacen comparaciones del alumnado por centros públicos y privados homogenizando el entorno familiar.
\end{abstract}

PALABRAS CLAVE: Educación secundaria, premio extraordinario, centros públicos, centros privados.

1. Educación Secundaria Obligatoria.

2. Ciencias Sociales, Geografía e Historia. 


\title{
COMPARATIVE ANALYSIS OF PUBLIC AND PRIVATE SCHOOLS MEASURED THROUGH THE EXTRAORDINARY PRIZE OF OSE ${ }^{3}$ IN GALICIA
}

\begin{abstract}
The aim of this article is to analyze the influence of the ownership of the centers and the family environment on the academic performance measured through the tests of the Extraordinary Prize of Secondary Education. Firstly, a review of the literature is made to let know the contribution of other authors regarding the influence of centers and other variables on the students' performance. Afterwards, based on the qualifications in all subjects (8) of the students that participated in the Extraordinary Prize of Secondary Education (1.522), the results were analyzed classifying them according to ownership of the center using an Unifactorial Analysis. Besides, it was analyzed how much influence does exist from the family environment on those results, measured through the education of parents and their purchasing power. The main results confirm that there are not significant differences regarding the ownership of the centers except in the subjects of "CSGH" and "Foreign language". Furthermore, it is proved the influence of family environment on the academic performance even though in "English" it is held in favor of private ownership when making comparisons between students of public and private centers homogenizing the family environment.
\end{abstract}

KEYWORDS: Secundary education, extraordinary prize, public schools, private schools.

Recibido: 19/04/2016

Aceptado: 13/07/2016

Correspondencia: Francisco Jesús Ferreiro Seoane, Universidad de Santiago de Compostela, Facultad de Derecho, Avda. Dr. Ángel Echeverri, s/n, Campus Vida 15782 Santiago de Compostela. Email: franciscojesus.ferreiro@usc.es.

\section{INTRODUCCIÓN}

La Consellería de Educación ${ }^{4}$ convoca todos los años desde el curso 2007-2008 los premios extraordinarios de ESO. El objetivo que se marca es otorgar un reconocimiento público al esfuerzo y dedicación del alumnado de esa etapa educativa, reforzar aquellos aspectos que inciden en la mejora del sistema educativo y juntar excelencia con equidad, igualdad de oportunidades y posibilidades de desarrollo personal. La educación se ha vuelto más accesible con los años, no obstante, se vuelve necesario realizar un análisis de la eficiencia de la formación educativa, finalidad que buscan los países de la UE (Jorge y Santín, 2010). Por ello, la Consellería de

3. Obligatory Secondary Education.

4. Es un organismo del gobierno regional de Galicia (Xunta de Galicia). 
Educación otorga 20 premios extraordinarios al rendimiento académico. El premio consiste en una dotación económica de 750 € para cada uno de los galardonados, un diploma acreditativo, y la distinción en el expediente académico. (Xunta de Galicia, $2012 a$ y 2012 b y 2013).

Aproximadamente se presentan a la prueba unos 350 discentes por año que tienen expedientes de sobresaliente de media en los cuatro cursos de la ESO, lo que equivale al 1,5\% del total de estudiantes de dicho nivel educativo en Galicia. Dado que se conceden 20 premios a los que se presentan, aproximadamente sólo el 6\% de los presentados con expedientes de sobresaliente obtienen la distinción.

El objetivo de este trabajo es analizar el rendimiento de los discentes que se han presentado al premio extraordinario de la ESO para el período 2008-2013, y analizar si existen diferencias entre el alumnado según el centro sea de propiedad pública o privada. Para ello, se analizará el resultado en todas las asignaturas y si es significativo. Por otro lado, se estudiará la posible influencia que pudiera tener el nivel socioeconómico y cultural de los padres en el rendimiento del discente y su grado de influencia.

Dado que la calidad es difícil de evaluar, se entiende que el rendimiento académico recoge la capacidad del sistema educativo de transmitir contenidos y/o competencias al alumnado y es una de las mediciones más utilizadas por cuanto posibilita la cuantificación, ya sea en forma de los resultados obtenidos en las diversas materias que forman parte del currículo, ya sea en pruebas nacionales de diagnóstico o en exámenes internacionales del estilo PISA (Programme for International Student Assessment), OCDE, TIMSS o PIRLS (Trends in International Mathematics and Science Study) de la IEA.

El informe del INCE (2013) (Instituto Nacional de Calidad y Evaluación) señala que la satisfacción que muestran los alumnos hacia el colegio es el factor fundamental en la valoración de su rendimiento escolar. A este respecto los estudios llevados a cabo por PISA (2009, 2011, 2012 entre otros) señalan que la titularidad del centro educativo es uno de los factores a tener en cuenta al analizar el rendimiento del alumnado. También se incluyen con especial énfasis en dichos informes, preguntas a los alumnos por su grado de satisfacción con el centro educativo y apuntan que, si se comparan los resultados educativos sin tener en cuenta los antecedentes socioeconómicos, los centros privados-concertados muestran unos resultados relativamente mejores que los de titularidad pública. Sin embargo, al introducir la variable del estatus socioeconómico y cultural, el impacto de la titularidad en algunos países disminuye o, incluso, desaparece. Según los últimos datos aportados por el informe PISA (2012), la media del alumnado que asiste a centros públicos en los países de la OCDE es casi del $85 \%$. El $15,1 \%$ se escolariza en centros privados independientes y concertados (subvencionados con fondos públicos). Dicho informe corrobora que el alumnado que asiste a centros públicos suele tener peor rendimiento en las evaluaciones que aquel que asiste a centros privados-concertados, pero matiza precisando que, si el contexto socioeconómico es similar en unos y otros centros, las diferencias en los resultados obtenidos no son significativas.

Numerosos estudios, además de otros factores, analizan si existen diferencias en el rendimiento académico en función de la titularidad de los centros educati- 
vos, como es el caso de Chubb y Moe (1990), cuyas evidencias de que los centros privados-concertados son mejores que los públicos avivan el debate. En esta línea se encuentran Calero et al. (2012), al afirmar que el rendimiento de los alumnos es superior en los centros de titularidad privada-concertada sobre los de titularidad pública, o Choi y Calero (2012), que afirman que este hecho se explica principalmente por las diferencias entre el perfil del alumnado escolarizado en los centros públicos y privados. Bien es cierto que las críticas no se hicieron esperar por la posible existencia de sesgos de selección del alumnado que provocan resultados no fiables en los centros privados. A este respecto, Witte (1992) no descubre evidencias suficientes que permitan confirmar diferencias significativas entre los rendimientos educativos entre unos centros y otros.

Por su parte, el Informe Coleman et al. (1966) concluye que los centros escolares por si solos tienen poca influencia en los logros académicos de los estudiantes y que existen otros inputs -características personales y familiares- más importantes en la determinación del output educativo. Así, Cordero et al. (2013), revela que una vez equiparado el nivel socioeconómico individual y el del centro, el rendimiento medio de los colegios públicos pasa a ser tan bueno como el de los privados en prácticamente todas las CCAA con muestra representativa.

Son interesantes las aportaciones de Bonal (2002), Sánchez (2008) o Villarroya (2001) al justificar las diferencias en el rendimiento académico de los discentes de los centros públicos por la inferior calidad de los recursos, el menor nivel socioeconómico y el mayor número de alumnos de origen inmigrante respecto a los privados. En esta dirección se encuentran los estudios de Calero y Waisgrais, (2009) y Calero et al. (2009), que ponen de manifiesto el inferior rendimiento del alumnado de origen inmigrante en comparación con los nacionales. Esta situación provoca que el sistema educativo -especialmente las instituciones públicas, pues es ahí donde se concentra la mayor parte del alumnado inmigrante- deba responder a las necesidades de los alumnos recién llegados, al ser uno de los principales instrumentos de integración de las personas de origen inmigrante (PISA, 2009). Perelman y Santín (2011) también cuestionan la superioridad de los centros privados alegando motivos como el "efecto composición" del alumnado escolarizado en unos y otros. Finalmente, Calero y Escardíbul (2007) concluyen que, por todo lo anteriormente expuesto, no existe una diferencia significativa en relación a la titularidad del centro.

Los estudios de Mancebón et al. (2007) centran este debate partiendo del trabajo realizado por Coleman et al. (1982) atendiendo al tipo de centro y concluyen que las escuelas privadas-concertadas son más eficaces que las públicas en la preparación del alumnado, incluso después de haber controlado las diferencias existentes en los antecedentes personales y socioeconómicos de los estudiantes de ambos tipos de centro.

El debate sobre si existen o no diferencias en el rendimiento académico teniendo en cuenta el tipo de centro ha suscitado numerosas investigaciones (Broc, 2006). No obstante, con respecto al alumnado objeto de nuestro estudio no existen evidencias de investigaciones que afirmen o nieguen nada en relación a la titularidad del centro educativo, ni al nivel socioeconómico o cultural.

Otras investigaciones, como las realizadas por Calero et al. (2007), Sánchez (2008) o García et al. (2008) aportan diversas justificaciones a las diferencias de ren- 
dimiento entre los adolescentes. Algunos confieren al autoconcepto y la motivación una mayor capacidad predictiva (Miñano y Castejón, 2008), otros dirigen sus estudios hacia las circunstancias personales, al origen del discente, a las familias, a las características socioeconómicas o a la motivación del alumnado hacia la escuela, como Cano (2000); Barca et al. (2004); Alonso Tapia (2005); Bermejo (2005); Woßmann, 2010; Cuetos y Domínguez, (2002) entre otros. A la hora de seleccionar el centro, los hogares utilizan a su vez otros factores que toman como indicadores de calidad educativa (Mancebón y Pérez, 2007; Alegre y Benito, 2012), tales como la orientación religiosa o pedagógica, la composición socioeconómica y/o étnica del alumnado o, en algunas comunidades autónomas (CCAA), los resultados publicados en los rankings, las infraestructuras o la difusión de otros servicios. Hemos de destacar la aportación de Kleinfeld et al. (1985), que indican que en el rendimiento del alumnado lo que influye son sus circunstancias personales y el hábito del alumno. García et al., 2014; se centran en que las características de los estudiantes (como la habilidad o la motivación), las de sus familias y el centro probablemente afecten, de forma simultánea, a la tasa de asistencia y al éxito.

Otros autores han estudiado la influencia de otras variables en el rendimiento académico, pudiendo haber una posible interacción entre ellas. Así Chao et al. 2015, afirman en sus estudios que existen diferencias significativas entre el alumnado del medio urbano y rural, siendo el primero el que muestra resultados más favorables. Chiswick y DebBurman, 2004, Meunier, 2011; afirman que la variable localización es significativa a la hora de explicar el rendimiento del alumnado siendo las diferencias más favorables en el entorno urbano que en el rural.

Otra variable que afecta al rendimiento académico según el Ministerio de Educación Cultura y Deporte (MECD) (2012), es el género, en línea con lo aportado por Backhoff et al., 2010 y Skolverket, 2006. Así Studenska, 2011; Cervini y Dari, 2009; Gaviria et al., 2004; Gipps y Murphy, 1994; MacCoby y Jacklin, 1974; indican que las alumnas tienen dotes superiores para las aptitudes verbales mientras que los alumnos destacan en el ámbito matemático. En cambio Casé et al., 2010; Fortoul et al., 2006; Nowell y Hedges, 1998; Pierart y Pavés 201; manifiestan que no existen dichas diferencias. Por otro lado, Ferreiro et al. (2014), concluyen que los chicos que se presentaron al premio extraordinario de la ESO obtuvieron mejor rendimiento que el género femenino.

\section{Método}

Una vez analizadas las diversas aportaciones de los autores en las que se pudo apreciar que existen variables que influyen en el rendimiento del discente y con conclusiones contradictorias, y tal como reflejan, Lobato y Alonso, (2002); Pascual, (2006); y Pena, et al. (2011), que existen diferencias de rendimientos en el alumnado, siendo necesario estudiar las distintas variables que influyen en ello, se procede a explicar el proceso metodológico seguido.

Se recuerda que el objetivo de este trabajo es averiguar si existen diferencias significativas entre el alumnado de los centros públicos y privados de Galicia en relación a las calificaciones de las diversas materias de los discentes que se presentaron al premio extraordinario de la ESO. Se estudia también si el nivel socioeconómico y cul- 
tural del entorno familiar de los estudiantes afecta también al éxito académico, pues pudiera ocurrir que un mayor rendimiento del alumnado de los centros en función de la titularidad pudiera estar motivada por otros factores como el entorno de la familia.

La muestra está formada por 1.522 estudiantes, de los cuales 940 pertenecen a centros públicos $(61,76 \%)$ y 582 a centros privados-concertados (38,24\%), que representan la totalidad del alumnado que se presentó a esta prueba para el período 2008-2013.

Las medidas utilizadas son las calificaciones obtenidas por el alumnado en dichas pruebas (nota media global, Lengua Extranjera, Lengua Castellana, Lengua Gallega, Matemáticas, Ciencias Sociales Geografía e Historia, Física y Química, Biología y Geología), clasificada según la titularidad del centro educativo (público o privado), las cuales se puede apreciar en la tabla 1.

Tabla 1. Notas medias por titularidad del centro (2008-2013)

\begin{tabular}{|c|c|c|c|c|c|c|c|}
\hline & L. Extranjera & L. Castellana & L. Gallega & CSGH & Matemáticas & Optativa & General \\
\hline 2008/09 & 7,72 & 5,91 & 6,55 & 4,35 & 5,48 & 4,59 & 5,77 \\
\hline Privada & 7,86 & 6,10 & 6,53 & 4,55 & 5,87 & 4,39 & 5,88 \\
\hline Pública & 7,64 & 5,80 & 6,57 & 4,24 & 5,26 & 4,71 & 5,70 \\
\hline $2009 / 10$ & 7,82 & 4,63 & 5,64 & 5,43 & 3,58 & 4,87 & 5,33 \\
\hline Privada & 8,01 & 4,56 & 5,78 & 5,66 & 3,78 & 5,00 & 5,46 \\
\hline Pública & 7,67 & 4,68 & 5,53 & 5,25 & 3,43 & 4,77 & 5,22 \\
\hline 2010/11 & 7,42 & 5,50 & 4,94 & 5,24 & 3,20 & 4,49 & 5,13 \\
\hline Privada & 7,58 & 5,54 & 4,98 & 5,45 & 3,30 & 4,60 & 5,24 \\
\hline Pública & 7,31 & 5,47 & 4,91 & 5,10 & 3,13 & 4,40 & 5,05 \\
\hline 2011/12 & 7,85 & 6,06 & 5,69 & 3,68 & 4,72 & 3,67 & 5,27 \\
\hline Privada & 8,34 & 6,27 & 5,67 & 3,81 & 4,88 & 3,60 & 5,42 \\
\hline Pública & 7,61 & 5,95 & 5,69 & 3,61 & 4,64 & 3,70 & 5,20 \\
\hline 2012/13 & 6,24 & 6,30 & 5,56 & 3,11 & 5,11 & 3,61 & 4,98 \\
\hline Privada & 6,54 & 6,03 & 5,04 & 2,79 & 4,77 & 3,57 & 4,79 \\
\hline Pública & 6,05 & 6,48 & 5,89 & 3,32 & 5,32 & 3,64 & 5,11 \\
\hline Total & 7,43 & 5,67 & 5,74 & 4,09 & 4,73 & 4,25 & 5,31 \\
\hline Privada & 7,67 & 5,64 & 5,64 & 4,88 & 4,13 & 4,26 & 5,37 \\
\hline Pública & 7,28 & 5,78 & 5,70 & 4,63 & 4,06 & 4,23 & 5,28 \\
\hline
\end{tabular}

Fuente: Elaboración propia a partir de datos de la Consellería de Cultura (Xunta de Galicia).

A continuación, se analizará la relación entre variables como la formación y el poder adquisitivo del entorno del discente con la titularidad del centro, para obtener esta información se ha procedido a realizar una encuesta a los colegios y a las familias del estudiantado presentado el premio extraordinario de la ESO.

Una vez clasificados los datos se ha procedido a realizar un Análisis de Varianza Unifactorial para comparar las medias en rendimiento entre los que estudian en un 
colegio de titularidad privada frente a uno público. Posteriormente se repite el análisis comparando el rendimiento de los discentes, pero homogenizando su entorno familiar. Todos los análisis efectuados se han realizado con el paquete estadístico IBM SPSS Statistics, versión 20.

\section{Resultados}

En la tabla 1, se puede observar cómo existe una tendencia positiva a favor del estudiantado de centros privados, con un resultado medio de 5,37, frente al 5,28 del estudiantado de los centros públicos y de medio global de 5,31. Este hecho llama la atención pues el alumnado que se puede presentar a las pruebas para optar al premio extraordinario de la ESO, han de tener una media de sobresaliente en toda la etapa secundaria obligatoria. Analizando los factores que explican la bajada en el resultado, hemos de mencionar los siguientes aspectos: en primer lugar, se trata de pruebas muy exigentes, dado que se otorgan 20 premios por año, lo que hace que solo el 6\% de los presentados obtengan el galardón, esto explica que hubiera discentes que en el examen de Física y Química hayan obtenido la nota de cero, si bien debemos destacar que en dicha prueba específica es donde se obtiene peor resultado (Ferreiro y Martínez, 2016). Un segundo hecho es que existe una diferencia de tiempo entre que se acaba la etapa de la ESO y la fecha de realización del examen, que suele ser en octubre del curso siguiente, por tanto, ha pasado un verano por medio. Un tercer factor está en que no todo el alumnado prepara la prueba, presentándose a ella debido a que no tiene coste, a pesar del atractivo de que suponga una mención especial en el expediente y se retribuye con $750 €$ a los ganadores. El resultado final es que las notas medias de Ciencias Sociales Geografía e Historia; Matemáticas y las optativas es de suspenso, siendo de aprobado en las asignaturas de lenguas (Extranjera, Castellana y Gallega).

En la misma tabla 1, se pueden apreciar que analizando las diferencias por centros, en las dos únicas asignaturas que se superan una diferencia de 0,25 puntos es en Lengua Extranjera y en $\mathrm{CSGH}$, mientras que en las otras es muy pequeña, si bien estos datos no permiten acreditar que sean significativas.

Para analizar si existe significatividad por titularidad de centro y asignaturas, se ha procedido a utilizar el método de Análisis unifactorial de comparación de muestras independientes, que se puede apreciar en la tabla 2. Nos confirma lo apreciado en la tabla 1, que en las únicas asignaturas en las que se aprecian diferencias significativas son inglés y CSGH, al tener una sig (bilateral) inferior a 0,05. Cumpliéndose parcialmente las conclusiones que autores como Mancebón et al. (2007), Sobrado, (2008), que partiendo del trabajo realizado por Coleman et al. (1982), indicaban que las escuelas privadas son más eficaces que las públicas en la preparación académica del alumnado.

Llegado a la conclusión que se producen diferencias significativas en dos asignaturas, debemos profundizar para ver en qué medida se pudiera dar una influencia del entorno socioeconómico en dicho rendimiento, como concluye (Ferreiro et al. 2016). Brenlla (2002), Encinas et al. (2009) afirmaban que existe una clara asociación entre el rendimiento académico y el nivel socioeconómico y profesional de los pro- 
genitores del alumnado. Cuetos y Domínguez (2002), siguiendo en la misma línea, consideran importante las características socioeconómicas y familiares del alumnado en el rendimiento académico, así como la motivación hacia la escuela. Castejón y Pérez, 1998; Estévez y Gutiérrez, 2002; y Rodrigo y Palacios,1998; afirman que los entornos familiares de nivel educativo medio o alto favorecen el rendimiento de los discentes, por lo que cuanto mayor es el nivel educativo de los padres, especialmente el de la madre, mayor incidencia positiva tendrá sobre el rendimiento académico y así, la estimulación educativa por parte de los padres con gran nivel sociocultural, provoca diferencias del rendimiento en estudiantes de distintos niveles económicos. Mullis et al., 2003 ratifican lo anterior destacando que el nivel educativo de los padres, el de renta y la presencia de un correcto ambiente en el hogar pueden estimular una mejora en el rendimiento académico de los estudiantes. Pelegrina et al., 2002 también siguen la idea anterior indicando que el afecto de los padres hacia el alumno se transforma en una alta competencia académica percibida y con la motivación hacia el cumplimiento académico.

Tabla 2. Estadísticas de grupo y muestras independientes por titularidad del centro

\begin{tabular}{|c|c|c|c|c|c|c|}
\hline Titularidad & & $\mathbf{N}$ & Media & $\begin{array}{c}\text { Desviación } \\
\text { estándar }\end{array}$ & $t$ & $\begin{array}{c}\text { Sig } \\
\text { (bilateral) }\end{array}$ \\
\hline \multirow{2}{*}{ Nota media } & Privada & 582 & 5,377 & 1,1346 & \multirow{2}{*}{1,426} & \multirow{2}{*}{, 154} \\
\hline & Pública & 940 & 5,290 & 1,1812 & & \\
\hline \multirow{2}{*}{$\begin{array}{l}\text { Nota } \\
\text { Inglés }\end{array}$} & Privada & 582 & 7,669 & 1,3299 & \multirow{2}{*}{5,449} & \multirow{2}{*}{,000 } \\
\hline & Pública & 940 & 7,279 & 1,3942 & & \\
\hline \multirow{2}{*}{$\begin{array}{c}\text { Nota } \\
\text { Castellano }\end{array}$} & Privada & 582 & 5,639 & 1,9274 & \multirow[b]{2}{*}{,- 560} & \multirow{2}{*}{, 576} \\
\hline & Pública & 940 & 5,695 & 1,8824 & & \\
\hline \multirow{2}{*}{$\begin{array}{l}\text { Nota } \\
\text { Gallego }\end{array}$} & Privada & 582 & 5,644 & 1,7640 & \multirow{2}{*}{$-1,481$} & \multirow{2}{*}{,139 } \\
\hline & Pública & 940 & 5,785 & 1,8362 & & \\
\hline \multirow{2}{*}{$\begin{array}{c}\text { Nota } \\
\text { Matemáticas }\end{array}$} & Privada & 582 & 4,134 & 2,5535 & \multirow{2}{*}{, 495} & \multirow{2}{*}{, 621} \\
\hline & Pública & 940 & 4,066 & 2,6236 & & \\
\hline \multirow{2}{*}{$\begin{array}{l}\text { Nota } \\
\text { CSGH }\end{array}$} & Privada & 582 & 4,880 & 1,7169 & \multirow{2}{*}{2,700} & \multirow{2}{*}{, 007} \\
\hline & Pública & 940 & 4,627 & 1,8533 & & \\
\hline \multirow{2}{*}{$\begin{array}{l}\text { Nota } \\
\text { Física-Química5 }\end{array}$} & Privada & 206 & 3,5461 & 2,20453 & \multirow{2}{*}{1,054} & \multirow{2}{*}{,292 } \\
\hline & Pública & 275 & 3,3287 & 2,26231 & & \\
\hline \multirow{2}{*}{$\begin{array}{c}\text { Nota } \\
\text { Biología-Geología }\end{array}$} & Privada & 180 & 4,1239 & 2,10995 & \multirow{2}{*}{,- 662} & \multirow{2}{*}{, 509} \\
\hline & Pública & 339 & 4,2540 & 2,14395 & & \\
\hline
\end{tabular}

Fuente: Elaboración propia a partir de datos de la Consellería de Cultura (Xunta de Galicia).

5. Es junto a Biología y Geología, las dos optativas mayoritariamente seleccionadas por el alumnado que suponen el $65,6 \%$. 


\section{Influencia de la formación de los padres en el resultado académico del alumnado}

En este apartado vamos a analizar en qué medida tanto la formación de los padres como el poder adquisitivo de los mismos pudiera estar influyendo en el mejor resultado obtenido en los colegios privados en las asignaturas de inglés y CSGH. Para ello se han definido las variables "estudios" y "profesiones" de la unidad familiar. En la variable "estudios" se han establecido 6 niveles (sin estudios, ESO, FP6, bachillerato, y universitarios).

Como podemos apreciar en la tabla 3, para el total de la muestra predominan las familias con el nivel formativo más elevado, es decir, con estudios universitarios, con un porcentaje de un $67,23 \%$. Lo que significa que los estudiantes que acuden a la prueba extraordinaria provienen de un entorno formativo elevado, bajando dicho porcentaje al 56\% en los centros públicos, y subiendo al $89 \%$ en los privados. Estos datos son sumamente importantes, al ser el nivel formativo de la unidad familiar una posible variable que pudiera guardar una relación significativa con las calificaciones de los alumnos.

Tabla 3. Nivel de estudios de la unidad familiar por titularidad

\begin{tabular}{cccc} 
& Público & Privado & Total \\
\hline UF Sin estudios & $4,58 \%$ & $1,22 \%$ & $3,40 \%$ \\
UF ESO & $12,42 \%$ & - & $8,09 \%$ \\
UF FP & $11,1 \%$ & $7,32 \%$ & $9,78 \%$ \\
UF Bachillerato & $16,34 \%$ & $2,44 \%$ & $11,49 \%$ \\
UF Universitarios & $55,56 \%$ & $89,02 \%$ & $67,23 \%$ \\
Total & $100,00 \%$ & $100,00 \%$ & $100,00 \%$ \\
\hline
\end{tabular}

Fuente: Elaboración propia a partir de datos de la Consellería de Cultura (Xunta de Galicia).

Para completar el análisis, se segmentan las notas de los alumnos cuyos padres son universitarios y acuden a centros privados frente a los que acuden a los públicos. Repetimos la operación con los hijos de los padres no universitarios, diferenciándolos también en función de la titularidad del centro a la que acuden los alumnos.

\section{Análisis de las asignaturas con diferencias significativas a favor de los centros privados (inglés y CSGH) y la formación del entorno}

En la tabla 4 se aprecia que el nivel formativo de la unidad familiar, con un Sig<0,05, es determinante y significativo en inglés y en CSGH a la hora de explicar las calificaciones de la nota media del alumnado. Por lo tanto, en la medida que coincide con la importancia de titularidad del centro, debemos seguir investigando si es el centro, el nivel formativo de los padres o las dos las que influyen en el resultado del alumnado.

6. Formación Profesional. 
Tabla 4. Rendimiento en función de la formación de la unidad familiar (2008-2013)

\begin{tabular}{|c|c|c|c|c|c|c|}
\hline & Padres & $\mathbf{N}$ & Media & $\begin{array}{c}\text { Desviación } \\
\text { estándar }\end{array}$ & $\mathbf{t}$ & $\begin{array}{c}\text { Sig. } \\
\text { (bilateral) }\end{array}$ \\
\hline \multirow{2}{*}{$\begin{array}{l}\text { Nota } \\
\text { Ingles }\end{array}$} & $\begin{array}{c}\text { No } \\
\text { universitarios }\end{array}$ & 385 & 6,853 & 1,5806 & \multirow{2}{*}{$-9,635$} & \multirow[t]{2}{*}{,000 } \\
\hline & Universitarios & 790 & 7,742 & 1,3384 & & \\
\hline \multirow{2}{*}{$\begin{array}{l}\text { Nota } \\
\text { CSGH }\end{array}$} & $\begin{array}{c}\text { No } \\
\text { universitarios }\end{array}$ & 385 & 4,012 & 1,8246 & \multirow[t]{2}{*}{$-5,794$} & \multirow[t]{2}{*}{,000 } \\
\hline & Universitarios & 790 & 4,802 & 2,111 & & \\
\hline
\end{tabular}

Fuente: Elaboración propia.

Para saber si la diferencia de calificaciones de los alumnos es debido al entorno cultural o al centro del que procede (los dos acreditaron significancia estadística) se estudia a continuación aquellos casos cuyos padres son universitarios y sus hijos acuden a centros públicos, frente a los que acuden a centros privados-concertados.

a) Análisis de las asignaturas con diferencias significativas a favor de los centros privados (Inglés y CSGH) cuando el entorno es de formación universitaria

Cuando se analizan los resultados de los estudiantes de los centros privados y públicos, con algún padre o madre con formación universitaria, tal como se refleja en la tabla 5, la puntuación media es mayor en los centros privados, en Lengua Extranjera con una significatividad de un $S i g=0,00<0,05$, el resto de materias no resultan significativas. Por tanto, en esta asignatura podemos concluir que, para el mismo grado de formación del entorno familiar, el centro privado sí obtiene mejor rendimiento en inglés.

Tabla 5. Estadísticas de grupo en que la unidad familiar del discente tiene formación universitaria

\begin{tabular}{ccccccc}
\hline & $\begin{array}{c}\text { Titularidad } \\
\text { centro }\end{array}$ & N & Media & $\begin{array}{c}\text { Desviación } \\
\text { estándar }\end{array}$ & t & $\begin{array}{c}\text { Sig. } \\
\text { (bilateral) }\end{array}$ \\
\hline Nota & Privada & 365 & 8,157 &, 9424 & 9,137 &, 000 \\
Ingles & Pública & 425 & 7,159 & 1,5407 & & \\
\hline Nota & Privada & 365 & 4,677 & 1,9955 & \multirow{2}{*}{, 857} &, 401 \\
CSGH & Pública & 425 & 4,656 & 2,2345 & & \\
\hline
\end{tabular}

Fuente: Elaboración propia.

b) Análisis de las asignaturas con diferencias significativas a favor de los centros privados (Inglés y CSGH) cuando el entorno no es de formación universitaria Se puede confirmar en la tabla 6 que se repiten los resultados anteriores, es decir, resultan beneficiados aquellos alumnos que estudian en centros privados frente a los públicos en la asignatura de Lengua Extranjera para un mismo nivel formativo de los padres. 
Tabla 6. Estadísticas de grupo en que la unidad familiar del discente no tiene formación universitaria

\begin{tabular}{ccccccc}
\hline & $\begin{array}{c}\text { Titularidad } \\
\text { centro }\end{array}$ & N & Media & $\begin{array}{c}\text { Desviación } \\
\text { estándar }\end{array}$ & t & $\begin{array}{c}\text { Sig. } \\
\text { (bilateral) }\end{array}$ \\
\hline $\begin{array}{c}\text { Nota } \\
\text { Ingles }\end{array}$ & Privada & 45 & 7,322 & 1,2584 & 2,662 &, 008 \\
\hline Pública & 340 & 6,656 & 1,6149 & & \multirow{2}{*}{, 416} \\
CSGH & Privada & 45 & 4,156 & 1,3013 & \\
\hline
\end{tabular}

Fuente: Elaboración propia.

\section{Influencia del nivel profesional de la familia en el resultado académico del alumnado}

En la variable "profesiones", después de manejar diferentes categorizaciones de muy diversa procedencia, se ha procedido a la siguiente clasificación:

- C1: Empresarios y directores de grandes y medianas empresas. Profesiones liberales. Militares o fuerzas armadas desde Comandante a General inclusive.

- C2: Empresarios de pequeñas empresas. Técnicos medios. Agentes comerciales, representantes y viajantes.

- C3: Empleados de oficina. Dependientes de comercio. Fuerzas armadas sin graduación.

- C4: Subalternos de oficina. Peones y obreros no cualificados de la industria. Personal de servicios domésticos.

Con respecto a la profesión de los padres se tiene que destacar que las diferencias se encuentran en la unidad familiar con mayor especialización profesional. Por ello hemos dividido las unidades familiares en dos grupos: C1 y "No C1" (C2, C3 y C4) del desglose efectuado anteriormente.

Los alumnos procedentes de una unidad familiar de alto grado laboral, es decir C1, se encuentran en mayor medida en los centros privado (tabla 7) con un 69,51\% frente a un $48,37 \%$ en los públicos. Se puede decir que en general los discentes que se presentan a las pruebas proceden de familias de nivel profesional elevado, repitiéndose casi la misma distribución que la concerniente al nivel de los estudios.

Tabla 7. Nivel profesional de la unidad familiar por titularidad

\begin{tabular}{cccc}
\hline & Público & Privado & Total \\
\hline C1 & $48,37 \%$ & $69,51 \%$ & $55,74 \%$ \\
No C1 & $51,63 \%$ & $30,49 \%$ & $44,26 \%$ \\
\hline
\end{tabular}

Fuente: Elaboración propia. 
A continuación, efectuamos el mismo proceso que el realizado anteriormente para el nivel de estudios, diferenciando los alumnos con padres pertenecientes al nivel $\mathrm{C} 1$ y no.

\section{Análisis de las asignaturas con diferencias significativas a favor de los centros privados (Inglés y CSGH) y el nivel profesional del entorno}

Se escogen las materias que en los análisis precedentes resultaban significativas a favor de los colegios privados. Se puede observar en la tabla 8 que los alumnos cuyos padres tienen un mayor poder económico reflejan un mayor rendimiento en Lengua Extranjera y CSGH, coincidiendo con la influencia de la titularidad, lo cual nos obliga a seguir analizando qué factor influye en el mejor rendimiento, si la titularidad del colegio o el nivel económico de los padres u ambas.

Tabla 8. Estadísticas de grupo en función del nivel profesional de la unidad familiar del discente

\begin{tabular}{|c|c|c|c|c|c|c|}
\hline & Padres & $\mathbf{N}$ & Media & $\begin{array}{c}\text { Desviación } \\
\text { estándar }\end{array}$ & $\mathbf{t}$ & $\begin{array}{c}\text { Sig. } \\
\text { (bilateral) }\end{array}$ \\
\hline \multirow{2}{*}{$\begin{array}{l}\text { Nota } \\
\text { Ingles }\end{array}$} & Trabajan como C1 & 654 & 7,778 & 1,3968 & \multirow{2}{*}{9,345} & \multirow{2}{*}{, 000 } \\
\hline & No trabajan como $\mathrm{C} 1$ & 520 & 6,991 & 1,5814 & & \\
\hline \multirow{2}{*}{$\begin{array}{l}\text { Nota } \\
\text { CSGH }\end{array}$} & Trabajan como C1 & 654 & 4,889 & 2,1018 & \multirow{2}{*}{6,112} & \multirow{2}{*}{,000 } \\
\hline & No trabajan como C1 & 520 & 4,194 & 1,9917 & & \\
\hline
\end{tabular}

Fuente: Elaboración propia.

Para saber si la diferencia de calificaciones de los alumnos es debido al nivel profesional de los padres o al centro del que procede, a continuación, analizamos aquellos casos cuyos padres pertenecen a un nivel laboral C1 y sus hijos acuden a centros públicos, frente a los que acuden a centros privados-concertados.

a) Análisis de las asignaturas con diferencias significativas a favor de los centros privados (Inglés y CSGH) cuando el entorno tiene el mayor nivel profesional (C1) Al considerar el mismo nivel socioeconómico de la familia, tal como se refleja en la tabla 9, la puntuación media es mayor en los centros privados. En concreto en Lengua Extranjera con una significatividad de un Sig=0,00<0,05. Por tanto, sí se puede concluir que la titularidad del centro es relevante en esta asignatura.

b) Análisis de las asignaturas con diferencias significativas a favor de los centros privados (Inglés y CSGH) cuando el entorno no tiene el mayor nivel profesional (C1)

Se confirma en la tabla 10 que resultan beneficiados aquellos alumnos (cuyos padres no trabajan en el nivel C1) que estudian en centros privados frente a los públicos en Inglés y CSGH. Podemos concluir que la titularidad del centro resulta importante en el alumnado con padres con peor nivel profesional. 
Tabla 9. Estadísticas de grupo en que la unidad familiar del discente trabaja en nivel C1

\begin{tabular}{ccccccc}
\hline & $\begin{array}{c}\text { Titularidad } \\
\text { centro }\end{array}$ & N & Media & $\begin{array}{c}\text { Desviación } \\
\text { estándar }\end{array}$ & t & $\begin{array}{c}\text { Sig. } \\
\text { (bilateral) }\end{array}$ \\
\hline $\begin{array}{c}\text { Nota } \\
\text { Ingles }\end{array}$ & Privada & 285 & 8,235 &, 9647 & 8,810 &, 000 \\
\hline Pública & 369 & 7,357 & 1,5008 & & \\
CSGH & Privada & 285 & 4,882 & 1,8483 &,- 088 &, 947 \\
\hline
\end{tabular}

Fuente: Elaboración propia.

Tabla 10. Estadísticas de grupo en que la unidad familiar del discente trabaja en nivel C1

\begin{tabular}{ccccccc}
\hline & $\begin{array}{c}\text { Titularidad } \\
\text { centro }\end{array}$ & N & Media & $\begin{array}{c}\text { Desviación } \\
\text { estándar }\end{array}$ & t & $\begin{array}{c}\text { Sig. } \\
\text { (bilateral) }\end{array}$ \\
\hline $\begin{array}{c}\text { Nota } \\
\text { Ingles }\end{array}$ & Privada & 125 & 7,600 & 1,1493 & 7,100 &, 000 \\
\hline Pública & 395 & 6,668 & 1,6211 & &, 003 \\
CSGH & Privada & 125 & 4,536 & 1,8820 & 3,047 & \\
\hline
\end{tabular}

Fuente: Elaboración propia.

Llegado a este punto donde se concluye que el centro tiene influencia positiva y significativa a favor de los discentes de la privada en la asignatura en Lengua Extranjera, debemos buscar las razones. Por un lado, existen colegios privados y concertados que su factor diferencial es precisamente una fuerte apuesta por los idiomas frente a la escuela pública. Esto se ve reforzado por la influencia de la formación de los padres (tabla 3) y que el $89,02 \%$ de los discentes de las escuelas privadas tienen un entorno familiar con formación universitaria que le otorga una gran importancia al aprendizaje de los idiomas extranjeros. Además, el 69,51\% de dicho entorno trabaja en profesiones de mayor nivel económico, lo que la facilita la financiación de intercambios, estancias en el extranjero o contratación de profesores particulares nativos para la mejora de los idiomas. Por otro lado, el nivel de profesores nativos presentes en la escuela pública es más bajo que en la escuela privada.

\section{DisCUSIÓN Y CONCLUSIONES}

Después de los análisis estadísticos donde se han analizado las variables, asignaturas (8), titularidad (público y privado), formación del entorno familiar (5 categorías) y entorno económico familiar (4 categorías), se pueden exponer las siguientes conclusiones. 
El alumnado que se presenta al premio Extraordinario cada año oscila entre 300 y 350, que han de tener una media de sobresaliente en toda la ESO y sólo el $6 \%$ de los presentados obtienen el premio, lo que indica la dificultad que supone conseguirlo.

Las notas medias obtenidas son sustancialmente bajas, pues siendo un colectivo de alumnado brillante, han tenido de media global 5,28 en los exámenes del premio extraordinario, siendo la media de suspenso en Matemáticas; Biología y Geología; Física y Química; y CSGH. Por el contrario, han aprobado en lenguas, siendo en inglés la mejor nota en todos los años. Las razones de este bajo rendimiento respecto a las notas obtenidas en su etapa de la ESO, son por un lado, la gran dificultad de las pruebas para obtener el premio; la diferencia de tiempo transcurrido entre la finalización de la ESO y la realización de la prueba, que suele ser en octubre del curso siguiente; y el que son exámenes para los que se tienen que preparar por su cuenta, siendo muy pocos los colegios (sobre todo privados) que ayudan al discente a afrontar dichas pruebas, de tal manera que muchos se presentan pues no supone coste alguno hacerlo y sin la debida preparación específica.

Otra conclusión es que en la mayoría de materias la nota más elevada corresponde a los alumnos procedentes de centros privados, siendo de manera estadísticamente significativa en las asignaturas de inglés y CSGH con respecto a los colegios públicos.

Por otro lado, se puede apreciar que es mayor el porcentaje de padres con formación universitaria en los colegios privados (89,02\%) que en los centros de titularidad pública (55,56\%). Algo similar, pero en menor cuantía, acontece cuando se analiza el nivel de categoría profesional de los padres, de tal manera que el 69,51\% del alumnado de los colegios privados tienen padres con el mayor nivel C1, el cual baja al $48,37 \%$ en los centros públicos.

También se ha podido verificar que el nivel de formación de los padres también tiene relevancia significativa en las notas de inglés y CSGH. Por ello se ha procedido a homogenizar los niveles formativos de los padres, de tal manera que cuando se comparan los alumnos de los centros privados y públicos cuyos padres tienen similar nivel educativo, se concluye que cuando son universitarios, se mantiene la diferencia significativa en inglés. Por tanto, se puede concluir que el alumnado de los colegios privados independientemente del nivel formativo de los padres, sí que obtiene mejores resultados de manera significativa en Lengua Extranjera.

Por otra parte, también el alumnado de procedencia de un entorno laboral superior obtiene mejores resultados académicos en relación a las restantes unidades familiares. Cuando se homogeniza por niveles profesionales de los padres del alumnado, cuando estos tienen un nivel profesional $\mathrm{C} 1$, se sigue manteniendo la diferencia significativa en la asignatura de inglés a favor de los centros privados. Y cuando el nivel profesional es distinto a C1, también se produce la significatividad en la otra asignatura CSGH, por tanto, una vez más los discentes de los centros privados sí obtienen mejores rendimientos al menos en inglés en todos los casos analizados.

Las razones de la influencia específica de los colegios privados en un mayor rendimiento en Lengua Extranjera derivan en que muchos de estos centros establecen su estrategia de fomento de los idiomas, con profesores nativos impartiendo dicha 
docencia, estancias en el extranjero, unido a que el perfil socioeconómico del entorno familiar de dicho alumnado es mayoritariamente de formación universitaria y mayor nivel económico que permite financiar este aprendizaje.

Estas conclusiones coinciden con el informe PISA (2012), Cordero et al. (2013), Bonal (2002), Sánchez (2008), Villarroya (2001), Calero y Waisgrais, (2009), Perelman y Santín (2011) y Calero y Escardíbul (2007) al afirmar que tan importante como el centro de estudio, es el nivel socioeconómico y cultural del entorno familiar del alumno. Si bien, en materias tan importantes como Lengua Extranjera se demuestra la importancia de la titularidad del centro, independientemente del entorno socioeconómico del discente.

Por último, se recomienda que se analizaran estos datos a futuro en la CCAA de Galicia para corroborar si se confirman estas conclusiones o cambian; además de hacer estudios en otras comunidades autónomas y otros países y poder contrastarlos con el análisis obtenido en Galicia.

\section{ReFERENCIAS BIBLIOGRÁFICAS}

Alegre, M. A. y Benito, R. (2012). ¿En qué se fijan las familias a la hora de escoger la escuela de sus hijos? Factores de elección y descarte escolar en la ciudad de Barcelona. Profesorado. Revista de curriculum y formación del profesorado, 16(3), 59-79. Recuperado de http://hdl.handle.net/10481/23100.

Alonso Tapia, J. (2005). Motivaciones, expectativas y valores - intereses relacionados con el aprendizaje: el cuestionario MEVA. Psicothema, 17(3), 404-411.

Backhoff, E., Sánchez, A., Peón, M. y Andrade, E. (2010). Comprensión lectora y habilidades matemáticas de estudiantes de educación básica en México: 20002005. Revista Electrónica de Investigación Educativa, 12(1), 2-18.

Barca, A., Peralbo, U. y Brenlla, J. C. (2004). Atribuciones causales y enfoques de aprendizaje. La escala Siacepa. Psicothema, 16(1), 94-103.

Bermejo, V. (2005). Microgénesis y cambio cognitivo: adquisición del cardinal numérico. Psicothema, 17(4), 559-562.

Bonal, X. (2002). El balance público-privado en el sistema de enseñanza español: Evolución y efectos sobre las desigualdades educativas. Educar, 29, 11-29.

Brenlla, M. (2002). Evaluación del bienestar psicológico en Iberoamérica. Argentina: Paidós.Goals?" Rural Development Perspectives, 13(3), 37-44.

Broc, M. A. (2006). Motivación y rendimiento académico en alumnos de Educación Secundaria Obligatoria y Bachillerato LOGSE. Revista de Educación, 340, 379-414.

Calero, J, Choi, A. y Waisgrais, S. (2009). Determinantes del rendimiento educativo del alumnado de origen nacional e inmigrante en PISA-2006. Cuadernos Económicos de ICE, 78, 281-310.

Calero, J. y Escardíbul, J. O. (2007). Evaluación de servicios educativos: el rendimiento en los centros públicos y privados medido en PISA-2003, Hacienda Pública Española, 83(4), 33-66.

Calero, J., Escardíbul, J. O., Waisgrais, S. y Mediavilla, M. (2007). Desigualdades socioeconómicas en el sistema educativo español. Madrid: CIDE-MEC. 
Calero, J. y Waisgrais, S. (2009). Factores de desigualdad en la educación española: una aproximación a través de las evaluaciones de PISA. Papeles de economía española, 119, 86-98.

Calero, J., Escardíbul, J. O. y Choi, A. (2012). El fracaso escolar en la Europa mediterránea a través de pisa-2009: radiografía de una realidad latente. Revista Española de Educación Comparada, 19, 69-103.

Cano, F. (2000). Diferencias de género en estrategias y estilos de aprendizaje. Psicothema, 12(3), 360-367.

Casé, L. R., Neer, R., Lopetegui, S. y Doná, S. (2010). Estrategias de aprendizaje y rendimiento académico según el género en estudiantes universitarios. Revista de Psicología, 11, 199-211.

Castejón, C. y Pérez, S. (1998). Un modelo causal-explicativo sobre la influencia de las variables psicosociales en el rendimiento académico. Revista Bordón. Sociedad Española de Pedagogía, 2(50), 170-184.

Cervini, R. y Dari, N. (2009). Género, escuela y logro escolar en matemática y lengua de la educación media: Estudio exploratorio basado en un modelo multinivel bivariado. Revista Mexicana de Investigación Educativa, 14, 1051-1078.

Chao, R., Ferreiro, F. y Mato, D. (2015). Análisis de las diferencias de rendimiento del alumnado de centros urbanos y no urbanos a partir de los resultados de los premios extraordinarios de educación secundaria obligatoria. Revista Electrónica Educare, 19(3). DOI: http://doi.org/10.15359/ree.19-3.237.

Chiswick, B. R. y Debburman, N. (2004). Educational attainment: analysis by immigrant generation. Economics of Education Review, 23, 361-379.

Choi, A. y Calero, J. (2012). Rendimiento académico y titularidad de centro en España. Profesorado. Revista de curriculum y formación del profesorado, 16(3), 31-57.

Chubb, J. E. y Moe, T. M. (1990). Politics, markets and the organization of schools. American Political Science Review, 82(4), 1066-1087.

Coleman, J., Hoffer, T. y Kilgore, S. (1982). High school achievement. Public, catholic and private schools compared. New York: Basic Books, Inc, Publishers.

Coleman, J., Campbell, E., Hobson, C., McPartland, J. y Mood, A. (1966). Equality of education opportunity. Washington: U.S. Office of Education.

Cordero Ferrera, J. M., Crespo Cebada, E. y Pedraja Chaparro, F. (2013). Rendimiento educativo y determinantes según PISA: Una revisión de la literatura en España. Revista de Educación, 362, 273-297.

Cuetos, F. y Domínguez, A. (2002). Efecto de la pseudohomofonía sobre el reconocimiento de palabras en una lengua de ortografía transparente. Psicothema, 14(4), 754-759.

Encinas, D., Díaz, C., Noriega, J. y Estrada, D. (2009). Locus de control y logro académico en dos tipos de ambiente de enseñanza para estudiantes universitarios. Pesquisas e Práticas Psicossociais, 3(2), 167-174. Recuperado de http://www. ufsj.edu.br/portal-repositorio/File/Encinas_etal.pdf.

Estévez, A., y Gutiérrez-Calvo, M. (2002). Context constraints, prior vocabulary knowledge and on-line inferences in reading. Psicothema, 14(2), 357-362. 
Ferreiro, F., Mato, D. y Chao, R. (2014). Análisis del rendimiento académico por género medido a través del premio extraordinario de la ESO. Revista de Investigaciones Políticas y Sociológicas (RIPS), 13(2), 1-24.

Ferreiro, F., Ríos, D. y Álvarez, D. (2016). Influencia del entorno familiar en el rendimiento académico en Galicia (España). Revista Iberoamericana de Educación, 70(1) (especial), 47-62.

Ferreiro, F. y Martínez, N. (2016). Influencia de las asignaturas optativas en el premio extraordinario de la Educación Secundaria Obligatoria (ESO). Revista RIPS, 15(1), $1-16$.

Fortoul, T. I., Varela, M., Ávila, M. R., López, S. y Nieto, D. M. (2006). Factores que influyen en los estilos de aprendizaje en el estudiante de medicina. Revista de la Educación Superior, 35(138), 55-62.

García, F. J., Rubio, M. y Bouachra, O. (2008). Población inmigrante y escuela en España: Un balance de la investigación. Revista de Educación, 345, 23-60.

García, J. I., Hidalgo, M. y Robles, J. A. (2014). Does grade retention affect students' achievement? Some evidence from Spain. Applied economics, 46(12), 13731392. Recuperado de https:/getinfo.tib.eu/de/suchen/id/zbwkat\%3A795560818/ Does-grade-retention-affect-students-achievement/.

Gaviria, J., Martínez, R. y Castro, M. (2004). Un estudio multinivel sobre los factores de eficacia escolar en países en desarrollo: El caso de los recursos en Brasil. Education Policy Analysis Archives, 12(20), 1-31. Recuperado de http://epaa.asu.edu/ epaa/consultado.

Gipps, G. y Murphy, P. (1994). A fair test: Assessment, achievement and equity. Buckingham: Open University Press.

Instituto Nacional de Calidad y Educación. INCE (2013). Recuperado de http://www. mecd.gob.es/inee/portada.html.

Jorge, J. y Santín, D. (2010). Determinantes de la eficiencia educativa en la Unión Europea. Hacienda Pública Española. Revista de Economía Pública, 193(2), 131-156.

Kleinfeld, J. S., Mcdiarmid G. W. y Hagstrom, D. (1985). Alaska's small rural high schools: Are they working? ISER Report Series, 22(3), 1-16.

Lobato, H. y Alonso, P. (2002). Elementos que influyen en el fracaso escolar. Huelva: Universidad de Huelva.

Maccoby, E. y Jacklin, C. (1974). The psychology of sex differences. Stanford: Stanford University Press.

Mancebón, M. J. y Pérez, D. (2007). Conciertos educativos y selección académica y social del alumnado. Revista de Economía Pública, 180, 77-106.

Mancebón, M. J., Martínez, N. y Pérez, D. (2007). Un análisis de la calidad percibida por los estudiantes en los centros públicos y privados de enseñanza secundaria. En XVI Jornadas de la Asociación de Economía de la Educación. Recuperado de http://works.bepress.com/domingoperez/4/.

Meunier, M. (2011). Immigration and student achievement: Evidence from Switzerland, Economics of Education Review, 30(1), 16-38.

Ministerio de Educación Cultura y Deporte (2012). Datos y Cifras curso escolar 2010/2011. Madrid: MECD. 
Miñano, P. y Castejón, J. L. (2008). Capacidad predictiva de las variables cognitivomotivacionales sobre el rendimiento académico. Revista Electrónica de Motivación y Emoción, 11, 1-13.

Mullis, R. L., Rathge, R. y Mullis, A. K. (2003). Predictors of academic performance during early adolescence. A contextual view. International Journal of Behavioral Development, 27(6), 541-548.

Nowell, A. y Hedges, L. (1998). Trends in gender differences in academic achievement from 1960 to 1994. Sex Roles, 39, 21-43. DOI: http://doi.org/10.1023/ A:1018873615316.

Pascual, B. (2006). Calidad, equidad e indicadores en el sistema educativo español. Pulso, 29, 43-58. Recuperado de http://dspace.uah.es/dspace/handle/10017/5147.

Pena, M., Extremera, N. y Rey, L. (2011). El papel de la inteligencia emocional percibida en la resolución de problemas sociales en estudiantes adolescentes. Revista Española de Orientación y Psicopedagogía, 22(1), 69-79.

Pelegrina, S., García, M. C. y Casanova, P. F. (2002). Parenting styles and adolescents' academic performance. Infancia y aprendizaje. Revista electrónica USAL, 25(2), 147-168.

Perelman, S. y Santín, D. (2011). Measuring educational efficiency at student level with parametric stochastic distance functions: an application to Spanish PISA results. Education Economics, 19(1), 29-49.

Pierart, G. y Pavés, F. (2011). Estilos de aprendizaje, género y rendimiento académico. Revista Estilos de Aprendizaje, 8(8), 71-84.

PISA 2009. OCDE (2010). Results: Overcoming Social Background: Equity in Learning Opportunities and Outcomes (Volume II). Paris: OCDE.

PISA 2011. MEC. Programa para la evaluación internacional de alumnos. OCDE. Informe español. Recuperado de http://www.educacion.gob.es/horizontal es/prensa/notas/2010/12/informe-pisa.htm.

PISA 2012. Programa para la Evaluación Internacional de los Alumnos. Informe español. Volumen I. Resultados y contexto. Recuperado de http://www.mecd.gob.es/ inee/estudios/pisa.html.

Rodrigo, M. J. y Palacios, J. (Coords.) (1998). Familia y desarrollo humano. Madrid: Alianza.

Sánchez, A. (2008). Efectos de la inmigración en el sistema educativo: el caso español. (Tesis doctoral). Universidad de Barcelona, Cataluña.

Skolverket, (2008). Gender differences in goal fulfilment and education choices. Swedish National Agency for Education, Stockholm: Skolverket.

Sobrado, L. M. (2008). Avaliación da calidade e Innovación dos Centros Educativos de Galicia. Santiago de Compostela: Universidade de Santiago de Compostela, Servizo de Publicacións.

Studenska, A. (2011). Educational level, gender and foreign language learning selfregulation difficulty. Procedia-Social and Behavioral Sciences, 29, 1349-1358. DOI: http://dx.doi.org/10.1016/j.sbspro.2011.11.373.

Villarroya, A. (2001). La financiación de los centros concertados. Madrid: MECDCIDE. 
Witte, J. F. (1992). Private school versus public school achievement: are there findings that affect the educational choice debate? Economics of Education Review, 11(4), 371-394.

Woßmann, L. (2010). Families, schools and primary-school learning: evidence for Argentina and Colombia in an international perspective. Applied Economics, 42, 2645-2665. DOI: http://doi.org/10.1080/00036840801964617.

Xunta de Galicia (2012a). DOG (Diario Oficial de Galicia). Orden del 3 de agosto de 2012 por la que se convocan los premios extraordinarios de educación secundaria obligatoria al rendimiento académico correspondientes al curso 2011/12. Recuperado de https://www.edu.xunta.es/premioseso.

Xunta de Galicia (2012b). Consellería de Cultura, Educación e Ordenación Universitaria: Información del Premio Extraordinario de la ESO 2012. Recuperado de https://www.edu.xunta.es/premioseso.

Xunta de Galicia (2013). DOG (Diario Oficial de Galicia). Orden del 22 de Julio del 2013 por la que se convocan los premios extraordinarios de educación secundaria obligatoria al rendimiento académico correspondientes al curso 2012/13. 